\title{
Distances appear different on hills
}

\author{
JEANINE K. STEFANUCCI, DENNIS R. PROFFITT, TOM BANTON, and WILLIAM EPSTEIN \\ University of Virginia, Charlottesville, Virginia
}

\begin{abstract}
When walking effort is increased due to manipulations such as wearing heavy backpacks, people perceive hills to be steeper and distances to be farther (Bhalla \& Proffitt, 1999; Proffitt, Stefanucci, Banton, \& Epstein, 2003). On the basis of these findings, we expected people to overestimate distances on steep hills relative to the same distances on flat ground, because of the increased effort required to ascend or descend them. This hypothesis is in contrast to the belief that distances are specified solely by optical and oculomotor information related to the geometry of the environment. To test the hypothesis, we investigated distance estimation on hills and flat terrains in natural and virtual environments. We found that participants judged steep uphill and downhill distances to be farther than the same distances on flat terrain. These results are inconsistent with the idea that spatial layout is perceived solely in terms of geometry, lending partial support to an effort hypothesis.
\end{abstract}

Just over 200 years ago, Meriwether Lewis and William Clark left their homes near Charlottesville, Virginia, to explore the uncharted western expanse of the North American continent. Clark, the cartographer of the 33-member company, drew maps of the lands over which they traveled. Study of these maps has revealed a systematic error that is of particular interest to us. Whenever the expedition traveled over rough or hilly terrain that made travel difficult, Clark overestimated the distances that had been traversed (Peebles, 1964). Today, we know that this is a general bias. People remember traversed extents to be greater if they are ascending or descending steep hills as opposed to walking on level terrain (Okabe, Aoki, \& Hamamoto, 1986).

Although these distortions may be due solely to memory biases, it is also possible that when viewing these extents, Clark perceived them to be greater when they were difficult to traverse. The present study supports this possibility. In five experiments, we showed that egocentric distances are perceived to be greater when people look at steep hills, either from the top or the bottom. In the case of estimating distances that are no longer in sight, memorial influences may lead to further distortion. In this vein, it is known that recalling the steepness of hills from memory leads to greater overestimations than judgments made when looking at them (Creem \& Proffitt, 1998).

That perceived extents on steep hills are overestimated introduces a geometrical paradox, because people's conscious awareness of the inclination of hills is itself overestimated (Bhalla \& Proffitt, 1999; Creem \& Proffitt, 1998;

\footnotetext{
We thank Maurice Hershenson, Mike Braunstein, and an anonymous reviewer for their comments on an earlier version of the manuscript. We also thank Mackenzie Carpenter, Jason Cervenka, Scott Early, Laura Lantz, Lauren Marsh, and Evan Rapoport for their help in collecting data. This research was supported by NSF ITR/Carnegie Mellon Grant 0121629 and ONR Grant N000140110060 to D.R.P. Correspondence should be addressed to J. K. Stefanucci, Department of Psychology, University of Virginia, 102 Gilmer Hall, Box 400400, Charlottesville, VA 22904-4400 (e-mail: jks8s@virginia.edu).
}

Proffitt, Bhalla, Gossweiler, \& Midgett, 1995). This overestimation of geographical slant is consistent, quite large, and is even observed when people's perspective provides a cross-sectional view of the incline (Proffitt, Creem, \& Zosh, 2001). With respect to the magnitude of this overestimation, observers typically report that $5^{\circ}$ hills have a slant of about $20^{\circ}$, and $10^{\circ}$ hills are typically judged to be about $30^{\circ}$

Given that people overestimate geographical slant, geometrical consistency demands that they should underestimate egocentric distances on the ground when hills are viewed from the bottom and overestimate egocentric distances when extents are judged from hilltops. To see why this is so, we examine the paradoxical case of viewing a hill from its base. Figure 1 depicts an observer, O, looking at a cone in two positions. First, there is a cone, $\mathrm{A}$, on flat terrain that is located distance $\overline{\mathrm{OA}}$ from the observer. ${ }^{1}$ Next, there is a cone, $\mathrm{B}$, located distance $\overline{\mathrm{OB}}$ from the observer on a $20^{\circ}$ hill. The apparent incline of the hill is overestimated to be $35^{\circ}$. Because the gaze angle to cone $\mathrm{B}$ is determined by its location on the actual hill, the cone's apparent location $\left(\mathrm{B}^{\prime}\right)$ on the overestimated hill moves it closer to the observer, and consequently its apparent egocentric ground distance should be compressed; the perception of distance $\overline{\mathrm{OB}}^{\prime}$ should be less than that perceived for distance $\overline{\mathrm{OA}}$. Thus, if observers estimate ground distances along the apparent incline in a manner that is consistent with the geometry of the apparent situation, they should underestimate the apparent distance to targets on hills relative to those on flat terrain.

An alternative to this geometrical account is one that appeals to the physiological effort that is associated with ascending a steep hill. Research has found that when ascending a hill, people must decrease their walking speed and lower their stride frequency in order to maintain the same metabolic cost as walking on flat terrain (Minetti, Ardigò, \& Saibene, 1993, 1994). Previously, we have shown that hills appear steeper when people are tired, encumbered by wearing a heavy backpack, in poor physical con- 


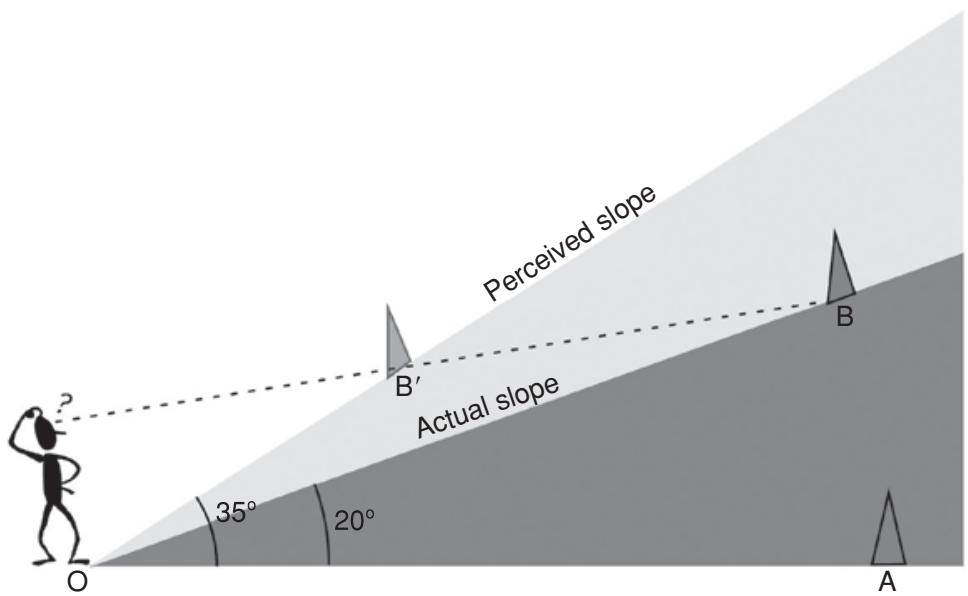

Figure 1. Cone $A$ on flat ground and cone $B$ on a hill are equidistant from the observer; distance $\overline{\mathrm{OA}}=\overline{\mathrm{OB}}$. The apparent incline of the hill is overestimated. The perceived location of $B\left(B^{\prime}\right)$ is determined by the line of sight, and thus, its location on the apparent hill is at $\mathbf{B}^{\prime}$. Geometrically, distance $\overline{\mathbf{O B}}^{\prime}$ is smaller than distance $\overline{\mathrm{OA}}$. In our experiments, distance $\overline{\mathrm{OB}}^{\prime}$ was perceived to be greater than the apparent distance of extent $\overline{\mathrm{OA}}$, which is inconsistent with a geometric representation of the distances.

dition, and elderly and in declining health (Bhalla \& Proffitt, 1999; Proffitt et al. 1995). Similarly, we have found that distances appear greater when people are encumbered by a backpack or have just walked on a treadmill, an experience that causes a visuomotor recalibration, which increases the amount of walking effort associated with traversing an extent (Proffitt, Stefanucci, Banton, \& Epstein, 2003). Generalizing these findings to the current situation of viewing a target on a steep hill, the observer would be expected to perceive its distance relative to the effort required to walk to it, and thereby overestimate its distance relative to viewing the cone on flat ground.

Our proposal that egocentric distances are perceived relative to anticipated effort introduces a geometrical inconsistency. The observer standing at the base of a steep hill would anticipate a greater walking effort when viewing the cone on the hill relative to viewing it on flat ground. By our account, apparent distance increases with anticipated effort, and thus, the cone would appear farther away when viewed on the hill, as opposed to the flat ground. If one applies this account to the situation depicted in Figure 1, the greater effort associated with walking up the hill relative to walking on flat terrain will cause distance $\overline{\mathrm{OB}}^{\prime}$ to be perceived as greater than that of $\overline{\mathrm{OA}}$, which is, of course, inconsistent with the geometry of the apparent situation. This account implies that observers do not perceive egocentric distance solely on the basis of the geometric properties inherent in optical stimulation. Instead, the perception of distance on an incline is a function of both the actual distance, as specified by optical variables, and the effort associated with traversing that distance. According to the effort hypothesis, distance estimates should be larger on hills than on flat ground.

In this article, we report five experiments examining perceived egocentric distances in both the real-world and virtual environments. Observers made distance judgments to targets on hills that were both steep and shallow. In some of the studies, viewing was from the hills' base, and in other studies it was from the top. Our motivation for using virtual reality was to assess a wider range of hills than is possible when testing is conducted outside. We first showed that people exhibit similar distance judgments in virtual reality as they do in the real world. Having done so, we used virtual environments as a means of presenting many different slopes to each participant. Overall, our results showed that perceived egocentric distance on steep hills is overestimated in a manner that is inconsistent with the geometry of the apparent spatial layout (see Figure 1).

\section{EXPERIMENT 1 Judgment of Distance on Flat and Uphill Terrains}

The purpose of this experiment was to test whether participants in a natural environment judged distances on hills to be different than the same distances on flat ground.

\section{Method}

Participants. Forty-three (18 male, 25 female) University of Virginia students participated in the experiment either as a requirement for an introductory psychology course or for compensation. Those who were paid received $\$ 10$ for their participation. All participants had normal or corrected-to-normal vision. They were naive about the purposes of the experiment and had not participated in previous distance studies in our lab.

Apparatus. The participants judged distances in an open, grassy field at the University of Virginia that was bounded on one side by a $20^{\circ}$ uphill slope (see Figure $2 \mathrm{~A}$ ). The distances were marked using golf tees that were not visible to the participants. The participants judged the distance from themselves to a small construction cone that was placed over a golf tee. During testing, each participant was given a 1-ft ruler to use as an aid for judging distances. After testing, 
A

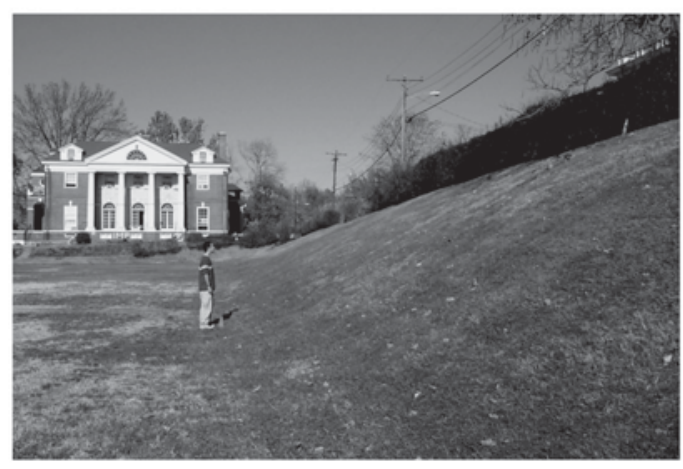

B Outdoor Distance Estimation

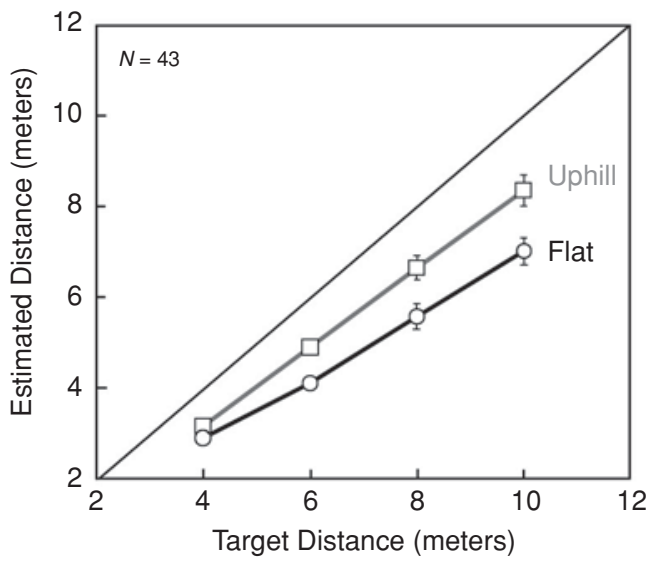

Figure 2. (A) The $20^{\circ}$ hill and flat terrain used in Experiment 1. (B) Results of Experiment 1: Distances were judged to be greater on a $20^{\circ}$ uphill slope than on flat terrain.

a tape measure was used to measure the participants' eye-heights in centimeters.

Design. The participants made 6 distance estimates in the flat ground and the uphill conditions, for a total of 12 distance estimates. In each condition, there were four target distances $(4,6,8$, and $10 \mathrm{~m})$ and two distractor distances $(1,2,12$, or $15 \mathrm{~m})$. Distractor estimates were not included in the analyses. Different distractor distances were used for the flat and uphill conditions in order to suggest to the participants that the distances were not identical across conditions. ${ }^{2}$ The presentation of distances was randomized, and the participants alternated between flat and uphill judgments.

Procedure. The participants were met by the experimenter away from the testing site so that they would not walk up or down the hill before testing. At the testing site, the participants were told that they would be estimating distances either on flat ground or up a hill. They were shown the cone and were told to judge the ground distance from themselves to the cone when it was placed at a distance. They were given the ruler and told that they could use it as a reference. All participants were asked to be as accurate as possible in their judgments and to produce verbal estimates in feet and inches. ${ }^{3}$

During testing, the participants were positioned at the base of the hill. They were asked to close their eyes and face the opposite direction from the cone being placed in the field. This ensured that the participants would not obtain familiar size cues from the experimenter as the cone was being positioned. The experimenter placed the cone in the field and walked back to the participants before they were allowed to open their eyes and turn around to judge the distance. They then gave a verbal estimate of the egocentric distance to the cone. The participants repeated this procedure for each cone distance, alternating between judging flat and uphill distances.

After completion of testing, the participants' eye-height was measured with a tape measure. The participants were also asked to stand at the base of the hill and estimate the steepness of the hill in degrees. They were then debriefed in full.

\section{Results}

A $2($ sex $) \times 2$ (order) $\times 2$ (terrain) $\times 4$ (distance) repeated measures ANOVA with distance and terrain as within-participants variables and sex and order as between-participants variables indicated main effects of terrain $[F(1,18)=35.01, p<.0001]$ and target distance $[F(3,16)=78.66, p<.0001]$. The participants judged uphill distances to be farther away than distances on flat ground. All distances were underestimated, as has been shown in previous studies (Amorim, Loomis, \& Fukusima, 1998; Loomis, Da Silva, Fujita, \& Fukusima, 1992; Norman, Todd, Perotti, \& Tittle, 1996), but participants underestimated the uphill distances less than these on flat ground (see Figure 2B).

As has been shown in previous studies, participants grossly overestimated the slant of the hill (Bhalla \& Proffitt, 1999; Creem \& Proffitt, 1998; Proffitt et al., 1995; Proffitt et al., 2001). The average participant verbally reported the $20^{\circ}$ hill to be $41^{\circ}$.

Eye-height was analyzed to determine whether participants were using gaze distance to make their judgments on the hill rather than ground distance. If gaze distance was a significant factor in target distance estimates, taller participants should have judged the target on the hill as being closer, resulting in a negative correlation with eye-height and target estimation. Contrary to this prediction, we did not find any significant correlations with eye-height and distance estimation. In addition, eye-height was not a significant predictor when added to the ANOVA performed above $[F(1,17)<0, p=.62]$, and so analyses in subsequent experiments did not include this factor.

\section{EXPERIMENT 2 \\ Judgment of Distance on Flat and Uphill Terrains in Virtual Reality}

In Experiment 2, we sought to replicate the results of Experiment 1 using a virtual environment. The purpose of the replication was to find out whether distance estimations in virtual reality would approximate those found in the real world.

\section{Method}

Participants. Twenty-four (12 male, 12 female) University of Virginia students participated in the experiment for psychology course credit. All participants had normal or corrected-to-normal vision and were naive about the purposes of the experiment. 
A

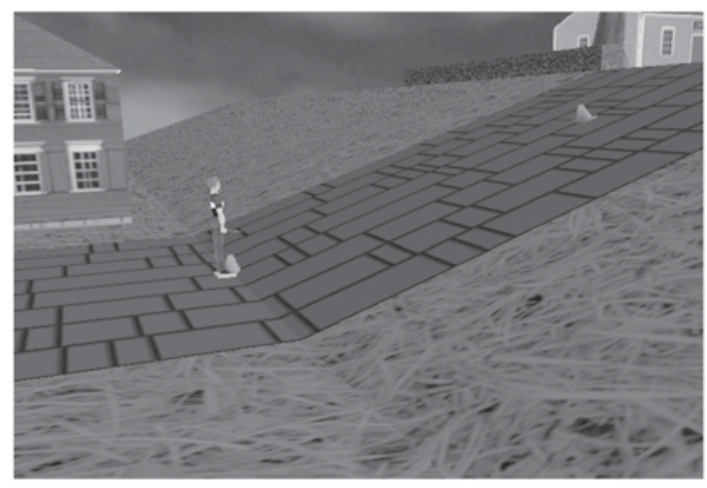

B Virtual Distance Estimation

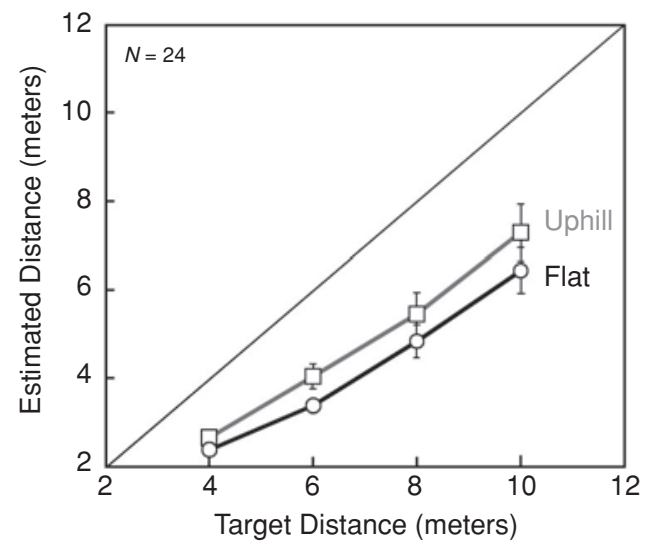

Figure 3. (A) Depiction of the $20^{\circ}$ hill in the virtual environment used in Experiment 2. The avatar represents the participant's viewpoint. (B) Results of Experiment 2: Distances viewed on uphill terrains were judged to be farther than distances viewed on flat terrain in virtual environments.

\begin{abstract}
Apparatus. While wearing a head-mounted display (HMD), the participants viewed a computer graphic rendering of a hill having the same slant as that used in Experiment 1 (see Figure 3A). The HMD was an n-Vision Datavisor with two active-matrix liquid crystal displays (LCDs) that operated in a VGA video format. The resolution of the LCDs was 640 (horizontal) $\times 480$ (vertical) pixels, by three color elements, and the field of view in each eye was $52^{\circ}$ diagonal. The images were presented stereoscopically (meaning each eye received an appropriately different perspective image) and refreshed at a rate of $60 \mathrm{~Hz}$. The images were viewed through collimating lenses that permitted the observer to focus at optical infinity. An Ascension SpacePad magnetic tracker was used to register the position and orientation of the HMD with $6^{\circ}$ of freedom. The end-to-end latency of the system (or the time it took for the computer to detect the position of the HMD and update the scene appropriately) was approximately $100 \mathrm{msec}$. The latency was calculated with the pendulum method (Liang, Shaw, \& Green, 1991). The virtual environment was created with Alice99 3-D software. ${ }^{4}$ To display the environment and register the position and orientation changes of the HMD, we used a PC with an Intel Pentium II processor, $128 \mathrm{MB}$ RAM, and an ATI Rage Pro Turbo graphics card.
\end{abstract}

Design. The design was the same as that used in Experiment 1.

Procedure. The participants wore the HMD and looked around the virtual world for a few seconds to get acclimated to the environment and the tracking. The participants were positioned with their feet next to a small orange cone. Another orange cone was placed in the distance, and they were asked to judge the ground distance from themselves to the cone. The participants also received a $1-\mathrm{ft}$ long virtual ruler that they could use as a reference while they made their judgments. A prop was held in their hands and was tracked to approximate the ruler in the virtual environment. They practiced estimating distance by making five judgments (from 1 to $15 \mathrm{~m}$ ) before testing began. No feedback was given during the practice estimation.

Flat and uphill extents were alternated. During test trials, the participants made a judgment and then closed their eyes while the experimenter recorded their responses. The virtual world was then changed to the inclination appropriate for the next trial, and the participants were asked to open their eyes and make another judgment. At the end of the trials, the participants were asked to judge the steepness of the hill in degrees before removing the HMD.

\section{Results}

The results of this study replicated those of Experiment 1 . A $2(\operatorname{sex}) \times 2$ (order) $\times 2$ (terrain $) \times 4$ (dis- tance) repeated measures ANOVA with distance and terrain as within-participants variables and sex and order as between-participants variables indicated main effects of terrain $[F(1,20)=8.877, p=.007]$ and target distance $[F(3,18)=40.072, p<.0001]$. The participants judged uphill distances to be farther away than the same distances on flat ground in the virtual environment (see Figure 3B).

The participants overestimated the slant of the virtual hill, judging the $20^{\circ}$ hill to be $34^{\circ}$ on average.

\section{EXPERIMENT 3 \\ Outdoor Distance Estimation Including Downhill Slopes}

Previous research has shown that a steep hill (greater than $25^{\circ}$ ) is judged as being steeper when one is standing at the top of the hill rather than at the base (Proffitt et al., 1995). The purpose of this experiment was to determine whether participants looking down a steep hill judged egocentric distance to be different from the same distance while viewing on flat ground or uphill.

\section{Method}

Participants. Eighteen ( 9 male, 9 female) University of Virginia undergraduates participated in the experiment for course credit in an introductory psychology course. All had normal or corrected-tonormal vision and were naive about the purposes of the experiment.

Apparatus. The participants judged distances on a $25^{\circ}$ hill near the University of Virginia bookstore (see Figure 4A). The flat terrain was a small, grassy area behind a dormitory near the hill. As in Experiment 1, the distances were marked using golf tees that were not visible to the participants. The participants used a 1-ft ruler as an aid while judging the distance from themselves to an orange cone placed on the terrain.

Design. The participants judged the perceived distance of a cone on flat, $25^{\circ}$ uphill, and $25^{\circ}$ downhill terrains. On each of the terrains, two lines of sight were used to minimize the use of fixed cues to distance. The participants changed their line of sight after each trial by moving to a new position. The two lines of sight were separated by $6 \mathrm{ft}$. We used a within-participants design with each person making esti- 
A

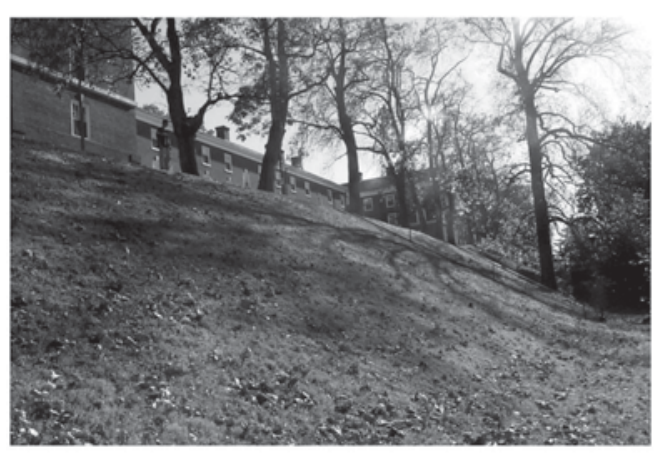

B Outdoor Distance Estimation

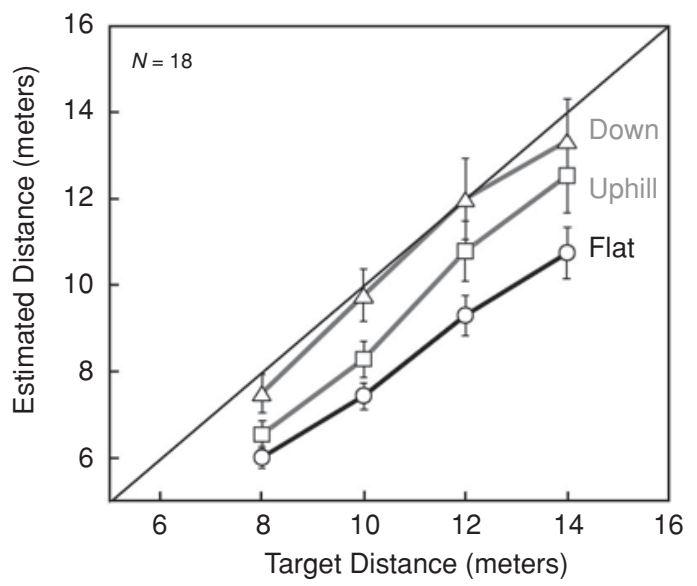

Figure 4. (A) The $25^{\circ}$ hill used in Experiment 3. (B) Results of Experiment 3: Distances viewed on steep downhill and uphill slopes were judged to be farther than the same distance viewed on flat terrain.

mates on all three terrains. The target distances were $8,10,12$, and $14 \mathrm{~m}$, along with two distractor distances randomly selected from one of three sets ( 1 and $6 \mathrm{~m} ; 2$ and $5 \mathrm{~m} ; 3$ and $4 \mathrm{~m}$ ). Distractors were grouped into sets so that the mean target distance in each block of trials was identical. The order of presentation of the type of terrain and the distractor group used for each block were counterbalanced, and the order of presentation of distances was randomized.

Procedure. The participants were greeted by the experimenter and taken to a flat, grassy area at the base of the hill to practice distance estimation before the test trials began. No feedback was provided. After practice, the participants were led to the appropriate area for their first set of judgments (either the base of the hill, the top of the hill, or the flat terrain). For each terrain, cones marked the starting points of the two lines of sight from which distance estimations were made. The participants alternated between the two starting points, which were $6 \mathrm{ft}$ apart, while making their judgments. The participants stood at the base of the cone, judged the ground distance to a target cone, and then walked to the other starting position, facing away from the test terrain to avoid inadvertently viewing the experimenter placing the target cone. When the experimenter had placed the cone for the next distance, the participants turned around to judge the distance.

The same procedure was followed for each terrain (flat, uphill, and downhill). At the end of the trials, the participants were led to the base of the hill to give a verbal estimate of the slant of the hill in degrees.

\section{Results}

A 2 (sex) $\times 6$ (order) $\times 2$ (block) $\times 3$ (terrain $) \times 4$ (distance) repeated measures ANOVA was performed with blocks of trials, terrain, and distance as within-participants variables and sex and order as between-participants variables. There were main effects of block $[F(1,6)=10.59$, $p=.017]$, terrain $[F(2,12)=35.589, p=.0001]$, and target distance $[F(3,18)=114.761, p=.0001]$. There were no effects of sex $(p=.238)$ or order $(p=.527)$. Post hoc analyses using Bonferroni correction revealed that participants judged distances as farthest on the downhill slope, somewhat closer on the uphill slope, and shortest on the flat terrain (see Figure 4B). The distance estimates were slightly longer in block 2 . In addition, participants' estimates of the slant of the $25^{\circ}$ hill averaged $50^{\circ}$.

\section{EXPERIMENT 4 Judgment of Distance on Flat, Uphill, and Downhill Terrains in Virtual Reality}

The purpose of this experiment was to explore distance estimation on hills with a variety of different slopes. Virtual reality was employed due to the practical impossibility of conducting such a study using real hills.

\section{Method}

Participants. Twenty-nine (14 male, 15 female) University of Virginia students participated in the experiment for introductory psychology course credit. All had normal or corrected-to-normal vision and were naive about the purposes of the experiment.

Apparatus. The virtual reality system used in this experiment was the same as that used in Experiment 2.

Design. The participants made five practice distance estimates (from 1 to $15 \mathrm{~m}$ ) outside before starting the experiment in virtual reality. No feedback was given for these practice estimates. In the virtual environment, the participants were asked to estimate six distances $(4,6,8,10,12$, and $15 \mathrm{~m})$ on hills of five different slopes (flat, $3^{\circ}, 6^{\circ}, 12^{\circ}$, and $24^{\circ}$ ). The participants were placed in either the uphill or downhill condition, meaning that they judged the distances on only one of two types of slopes along with the flat terrain. Each of the six distances was presented once on each degree of slope for a total of 30 distance estimates.

Procedure. The participants were led outside to practice making distance estimates in the real world before testing. Each participant was given a 1-ft ruler as a reference and stood at a cone to make the estimates. While the target cone was being placed, the participants faced away from the testing area. After placement, the participants turned around and estimated the egocentric ground distance to the cone in feet and inches, being as accurate as possible. The participants made five practice estimates on flat terrain before going back inside for the test trials in virtual reality.

After donning the HMD, the participants were given a few minutes to familiarize themselves with the virtual world. If they were judging uphill slopes, they found themselves on a village street at the base of a hill with flat ground behind them. If they were judging downhill slopes, they were positioned at the top of the same hill with flat ground behind them. They were given a $1-\mathrm{ft}$ virtual ruler to refer to while making their distance judgments. They aligned themselves 


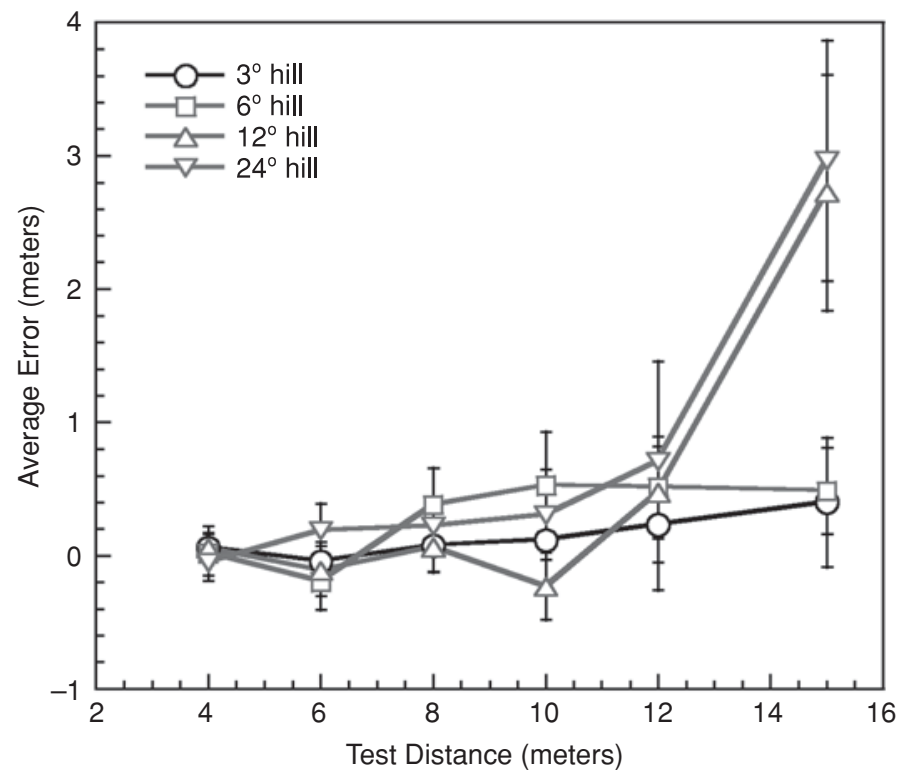

Figure 5. Difference scores are shown for distance estimates made on downhill and uphill slopes in virtual reality (collapsing across uphill and downhill conditions). A score of 0 represents no difference between distance estimates made on hilly and flat terrains. The greatest difference was found for the longest target distances on the steeper hills, suggesting that the perceived effort involved in ascending or descending these hills made the distances appear bigger.

with the small virtual cone at their feet and closed their eyes. A virtual target cone was placed on the virtual terrain. The participants opened their eyes and gave their distance estimate in feet and inches. They then turned $180^{\circ}$ and estimated the distance along flat ground. They alternated between flat and sloped conditions for all test trials.

\section{Results}

For each participant, we calculated a difference score for each slope and distance combination, consisting of the reported distance on the hill minus the reported distance on flat ground. A 2 (uphill or downhill) $\times 4$ (degree of slope) $\times$ 6 (distance) ANOVA was run on the difference scores with the first factor as between participants and the others as within participants. The analysis revealed main effects of degree of slope $[F(3,75)=3.50, p=.019]$ and distance $[F(5,135)=3.827, p=.003]$. Post hoc analyses using Bonferroni correction showed that the difference scores for the $3^{\circ}$ hill were significantly different from those for the $12^{\circ}$ and $24^{\circ}$ hills (see Figure 5). On the $3^{\circ}$ and $6^{\circ}$ hills, distance estimates were the same as on flat ground. On the $12^{\circ}$ and $24^{\circ}$ hills, distances were judged to be greater, especially at the 12- and 15-m distances. There were no betweenparticipants main effects of condition (uphill vs. downhill judgments, $p=.61)$ or gender $(p=.88)$.

\section{EXPERIMENT 5 \\ Judgment of Distance on Shallow Downhill Slopes}

According to the effort hypothesis, apparent distance is related to anticipated walking effort. This account sug- gests that a shallow downhill slope might make targets appear closer, because it should be slightly easier to descend a gradual incline than to traverse flat ground. This decrease in apparent distance was not found on the shallow $3^{\circ}$ virtual hill in the previous experiment. In case a subtle effect was missed due to the use of a virtual environment, we conducted a similar experiment using a real shallow downhill slope to determine whether distance perception might be underestimated in this condition.

\section{Method}

Participants. Twenty-four (12 male, 12 female) University of Virginia students participated in the experiment either for course credit or for compensation. Those who were paid received $\$ 10$ for their participation. All participants had normal or corrected-tonormal vision and were naive about the purposes of the experiment.

Apparatus. The participants judged distances in a flat, open, grassy field at the University of Virginia and on a hill that sloped down $3^{\circ}$. As in the other experiments, distances were marked so as not to be visible to the participant. A cone served as the target, and a 1 -ft ruler was given for reference during the test trials.

Design. The design of this experiment was the same as that of Experiment 3.

Procedure. The procedure of this experiment was the same as that of Experiment 3. However, the participants made estimates on the shallow downhill slope and flat terrain only.

\section{Results}

A 2 (sex) $\times 2$ (order) $\times 2$ (block) $\times 2$ (terrain) $\times 4$ (distance) repeated measures ANOVA was performed with blocks of trials, terrain, and distance as within-participants variables, and sex and order as between-participants variables. There were main effects of block $[F(1,20)=11.81$, 
$p=.003]$ and target distance $[F(3,18)=27.710, p<$ $.0001]$. There were no effects of $\operatorname{sex}(p=.19), \operatorname{order}(p=$ $.42)$, or terrain $(p=.28)$. Distance estimates increased from block 1 to block 2 and as target distances increased. However, the downhill distance estimates were not different from the flat ground estimates. On average, the participants verbally estimated that the $3^{\circ}$ downhill slope appeared to slope downward by $6.3^{\circ}$.

\section{DISCUSSION}

Across multiple experiments, our findings showed that people overestimated egocentric distances on steep uphill and downhill terrains relative to the same distances on flat terrain. These findings point to a lack of internal consistency in the geometry of perceived spatial layout. For apparent slant and distance to be geometrically consistent, the targets viewed from the base of a hill should appear nearer than those seen on flat ground because the slant of hills is grossly overestimated (Figure 1). Our data clearly do not follow this pattern.

Experiments 1 and 2 showed that participants overestimated distances on a $20^{\circ}$ uphill slope (relative to estimates on flat ground), both in the real world and in virtual reality. In accord with previous studies, participants also overestimated the slant of the hill (Bhalla \& Proffitt, 1999; Creem \& Proffitt, 1998; Proffitt et al., 1995; Proffitt et al., 2001). These concomitant overestimations of slant and distance are geometrically inconsistent.

In Experiment 3, participants overestimated distances on a $25^{\circ}$ hill (both uphill and downhill) relative to their estimates of the same distances on flat ground. This grassy hill is very difficult to ascend and impossible to descend without falling or breaking into a run. We believe that the effort associated with walking down the steep hill influenced participants' judgments of distance, but we acknowledge that the present studies did not directly test this assertion. Overestimating the downhill distances is consistent with the geometry and effort hypotheses; however, overestimating the uphill distances is only consistent with the effort hypothesis, leading us to favor the effort hypothesis as more parsimonious with this result.

Experiment 4 employed a wider range of hill slopes in a virtual environment. Both uphill and downhill distance judgments were overestimated relative to estimates on flat terrain, but only when the hills were fairly steep. Whether distance perception is, in fact, only influenced by steep hills cannot be concluded from this study because the effect could be due to the peculiarities of virtual environments. For this reason, we conducted Experiment 5, in which we measured distance estimation on a shallow $\left(3^{\circ}\right)$ downhill slope in the real world. We did not find a difference between distance estimates on flat terrain and on the shallow slope.

We expected distances on shallow hills to look closer than those on flat terrain, given that it should be easier to walk to a target on a gradual downhill incline, but our data did not support that prediction. It may have been that the metabolic energy required to walk down the shallow hill was not sufficiently different from that needed to traverse the flat terrain, thereby making it hard to find a difference in distance perception due to effort. Moreover, we acknowledge that the experiments presented here were not direct tests of the effort hypothesis. Perhaps future studies could include a direct manipulation of effort such as encumbering participants with a heavy backpack or asking them to throw a heavy ball to a target on the hill (Bhalla \& Proffitt, 1999; Proffitt et al., 2003; Witt, Proffitt, \& Epstein, 2004). Likewise, it is evident that we will need to conduct studies that would increase our understanding of effort as a concept and help to further define this variable. In this vein, including metabolic measures such as oxygen intake or heart rate would be useful, because they ought to increase with effort manipulations (e.g., wearing a heavy backpack) and may be correlated with measures of perceived distance and slant. Despite the lack of metabolic indicators, it is evident that any account that preserves a geometrical consistency between perceived distance and slant cannot explain all of our data.

Another important consideration is whether our measure (verbal report of distance) is a valid indicator of perceived distance. Verbal reports have been criticized in the past because they may reflect cognitive (not perceptual) judgments of egocentric distance. The concern is that participants might bias their judgments on the basis of their thoughts or reasoning about the experiment. By this account, our participants may have overestimated distances on hills relative to flat ground because their judgments, as opposed to their perceptions, were influenced by the experimental manipulations.

Allaying this concern, two bodies of evidence suggest that verbal reports are a valid indicator of perceived egocentric distance in the context of our studies. The first comes from previous work conducted by other members of our research group. Witt et al. (2004) showed that manipulations of effort affected not only verbal reports, but also a perceptual matching measure of distance (see their Experiment 2). In addition, they showed that when anticipated walking effort was increased, a visually guided action measure (i.e., blindwalking) was affected (Witt, Proffitt, \& Epstein, 2005). Second, and more generally, Philbeck and Loomis (1997) found that when measuring the perception of egocentric distance there is no dissociation in verbal reports and blindwalking (in contrast to slant estimates, where verbal reports are overestimated but visually guided actions are accurate). In their studies, a reduced-cue environment affected both measures of distance (verbal reports and blindwalking) in equal proportion. On the basis of these findings, we believe that our measure of perceived distance is indicative of participants' underlying perceptions and is not the result of cognitive corrections in their judgments.

As an alternative to our effort hypothesis, one could attribute our results to differences in the optical information available on hills relative to flat ground. For example, distance judgments could be influenced by texture gradient compression that differs on hills relative to the flat ground. By such an account, the overestimation of distances on 
steep hills could be due to differences in texture gradient compression rather than differences in the effort needed to ascend or descend these hills. However, even if this were the case, the relevant optical variables are not being processed so as to preserve geometrical consistency between perceived distance and slant.

The paradoxical finding that participants overestimate distance, given that they also overestimate slant, is an inconsistency that should be further explored. It should be noted that finding geometrical inconsistencies in perception is not a unique occurrence. Related deviations have been found in earlier studies on perceiving spatial layout (Epstein, 1977; Epstein, Park, \& Casey, 1961; Sedgwick, 1986). Such findings challenge standard accounts of perception, which typically assume that relationships among percepts will mirror geometric relationships to some degree. Two familiar instances of geometrical inconsistency, involving size, distance, and motion, are observed in the moon illusion and the trapezoidal window S-motion demonstration (Hershenson, 1999, pp. 123-130). The presence of geometrical inconsistency, however, is not limited to conditions of illusory perception. For instance, Gillam $(1967,1995)$ reported an inconsistency involving shape, slant, and binocular disparity, which is analogous to the better known size-distance paradox. When one of the two binocular images is magnified by the interposition of an aniseikonic lens, under certain conditions one obtains distortions of perceived shape in the geometrically predicted direction, while at the same time, the effect on perceived slant is opposite to that predicted by geometry.

Although inconsistencies of this sort have been recognized for several decades, their theoretical implications remain unresolved. One view, promoted by Gogel (1990), attributes the inconsistencies to the intrusion of cognitive factors in the response stage. Adopting this view allows the premise of geometric consistency, which underlies most claims for perceptual interdependency (e.g., the family of invariance hypotheses), to remain intact. An example is Gogel and Mertz's (1989) treatment of the size-distance paradox manifested in the moon illusion. Another view, toward which one of us inclines (Epstein \& Rogers, 2003), is that these seeming inconsistencies are a consequence of the modular organization of the system underlying perceived spatial layout. According to this conception, percepts (e.g., size and distance, lightness and slant), which the standard conception takes to be unexceptionally interdependent, are in fact independently processed. Since the present experiments were not designed to assess these options, or any other, our findings do little to clarify the matter, but rather highlight the nature of the problem that needs resolution.

\section{CONCLUSIONS}

Our previous research has shown that perceiving the basic geometrical properties of the ground-orientation and extent - is influenced by the effort required to traverse the terrain in question. Apparent slant and flat ground distances are overestimated following experimental ma- nipulations that increase walking effort (Bhalla \& Proffitt, 1999; Proffitt et al., 1995; Proffitt et al., 2003).

The present study showed that distances on steep hills are also overestimated, which introduces a geometrical inconsistency in distance perception. It is, however, our contention that perceiving spatial layout entails more than a geometrical analysis of relevant optical variables. The geometrical properties of optical information are, of course, the primary determinants of perceived layout; however, this information may be modulated by the effort associated with acting on the layout. We contend that spatial perception combines a specification of environmental affordances with the energetic costs associated with relevant behaviors.

\section{REFERENCES}

Amorim, M.-A., Loomis, J. M., \& Fukusima, S. S. (1998). Reproduction of object shape is more accurate without the continued availability of visual information. Perception, 27, 69-86.

Bhalla, M., \& Proffitt, D. R. (1999). Visual-motor recalibration in geographical slant perception. Journal of Experimental Psychology: Human Perception \& Performance, 25, 1076-1096.

Creem, S. H., \& Proffitt, D. R. (1998). Two memories for geographical slant: Separation and interdependence of action and awareness. Psychonomic Bulletin \& Review, 5, 22-36.

EPSTEIN, W. (1977). Stability and constancy in visual perception: Mechanisms and processes. New York: Wiley.

EPstein, W., PARK, J., \& CASEY, A. (1961). The current status of the size-distance hypotheses. Psychological Bulletin, 58, 491-514.

EPStein, W., \& Rogers, S. (2003). Percept-percept couplings revisited. In U. Savardi \& A. Mazzocco (Eds.), Figura e sfondo (pp. 93-106). Padua: CLEUP.

Gillam, B. (1967). Changes in the direction of induced aniseikonic slant as a function of distance. Vision Research, 7, 777-783.

GiLlam, B. (1995). The perception of spatial layout from static optical information. In W. Epstein \& S. Rogers (Eds.), Perception of space and motion (pp. 23-67). San Diego: Academic Press.

GoGEL, W. C. (1990). A theory of phenomenal geometry and its applications. Perception \& Psychophysics, 48, 105-123.

Gogel, W. C., \& Mertz, D. L. (1989). The contribution of heuristic process to the moon illusion. In M. Hershenson (Ed.), The moon illusion (pp. 235-258). Hillsdale, NJ: Erlbaum.

Hershenson, M. (1999). Visual space perception: A primer. Cambridge, MA: MIT Press.

Liang, J., ShaW, C., \& GreEN, M. (1991). On temporal-spatial realism in the virtual reality environment. Proceedings of the Association for Computing Machinery: Symposium on User Interface Software and Technology, 4, 19-25.

Loomis, J. M., Da Silva, J. A., Fujita, N., \& Fukusima, S. S. (1992). Visual space perception and visually directed action. Journal of Experimental Psychology: Human Perception \& Performance, 18, 906-921.

Minetti, A. E., Ardigò, L. P., \& SAibene, F. (1993). Mechanical determinants of gradient walking energetics in man. Journal of Physiology, 472, 725-735.

Minetti, A. E., Ardigò, L. P., \& Saibene, F. (1994). The transition between walking and running in humans: Metabolic and mechanical aspects at different gradients. Acta Physiologica Scandinavica, 150, 315-323.

Norman, J. F., Todd, J. T., Perotti, V. J., \& Tittle, J. S. (1996). The visual perception of three-dimensional length. Journal of Experimental Psychology: Human Perception \& Performance, 22, 173-186.

Oкаве, A., Aoki, K., \& Намамото, W. (1986). Distance and direction judgment in a large-scale natural environment: Effects of a slope and winding trail. Environment \& Behavior, 18, 755-772.

Peebles, J. J. (1964). Rugged waters: Trails and campsites of Lewis and Clark in the Salmon River country. Idaho Yesterdays, 8, 2-17.

Philbeck, J. W., \& Loomis, J. M. (1997). Comparison of two indicators of perceived egocentric distance under full-cue and reduced-cue 
conditions. Journal of Experimental Psychology: Human Perception \& Performance, 23, 72-85.

Proffitt, D. R., Bhalla, M., Gossweiler, R., \& Midgett, J. (1995). Perceiving geographical slant. Psychonomic Bulletin \& Review, 2, 409-428.

Proffitt, D. R., Creem, S. H., \& Zosh, W. D. (2001). Seeing mountains in mole hills: Geographical-slant perceptions. Psychological Science, 12, 418-423.

Proffitt, D. R., Stefanucci, J., Banton, T., \& Epstein, W. (2003). The role of effort in perceiving distance. Psychological Science, 14, 106112.

SEDGWICK, H. A. (1986). Space perception. In K. R. Boff, L. Kaufman, \& J. P. Thomas (Eds.), Handbook of perception and human performance: Vol. 1. Sensory processes and perception (pp. 21.1 to 21.57). New York: Wiley.

Witt, J. K., Proffitt, D. R., \& Epstein, W. (2004). Perceiving distance: A role of effort and intent. Perception, 33, 577-590.

Witt, J. K., Proffitt, D. R., \& Epstein, W. (2005). The role of effort and intention in distance perception [Abstract]. Journal of Vision, 3, 15a.

\section{NOTES}

1. The apparent egocentric distance to this cone would likely be underestimated (Amorim, Loomis, \& Fukusima, 1998; Loomis, Da Silva, Fujita, \& Fukusima, 1992; Norman, Todd, Perotti, \& Tittle, 1996).

2 . The distractor distances across the uphill and flat conditions were also different because the extent of the hill was not great enough to be able to place distractors at long distances. Therefore, the distractor distances on the hill were placed at $1 \mathrm{~m}$ and $2 \mathrm{~m}$, but the distractor distances on the flat ground were placed at $12 \mathrm{~m}$ and $15 \mathrm{~m}$. This discrepancy raises a concern about participants' regressing their distance estimates to different mean distances in the two conditions. However, it is important to note that, based on the chosen distractors, the mean distance is lower in the uphill condition than in the flat condition. If the mean of the distances in each condition affected the participants' estimates, estimates of perceived distance on the flat terrain should be greater than those on the hill. However, we found that estimates on flat terrain were less than those on the hill. For this reason, we do not believe that a compression to the mean affected distance estimates to the targets. This potential confound was controlled for in subsequent experiments.

3. Previous research in our lab and others has used perceptual matching and blindfolded walking as converging measures of apparent distance (Loomis et al., 1992; Witt, Proffitt, \& Epstein, 2004). However, the steep incline of the experimental hill made perceptual matching and blindfolded walking impossible to employ. Similar studies have shown that perceptual matching measures are consistent with verbal reports (Witt et al., 2004).

4. Alice 99 was created by the Stage 3 Research Group at Carnegie Mellon University and is available for free at www.alice.org.

(Manuscript received March 4, 2004; revision accepted for publication December 6, 2004.) 